\title{
The Method of Determinant Value Calculation Using Eigenvalues
}

\author{
Hongling Meng ${ }^{1, a^{*}}$, Kaiguang Zhang ${ }^{1, b}$, Mingting $\mathrm{Ba}^{1, \mathrm{c}}$ \\ ${ }^{1}$ Zhengzhou Normal University, Zhengzhou,Henan, China \\ ahnmhl@126.com, bzzgis@sina.com, cbmt1234@126.com
}

Keywords: Covariance Matrix, Linear Transformation, Eigenvalue, Determinant

Abstract. The paper utilizes the linear transformation in the analysis of covariance matrix of observed values about natural phenomenon, while computing eigenvalues, the corresponding determinant value is calculated, at last the relationship between the eigenvalues and determinant value is given.

\section{Introduction}

Statistical analysis is an important method for understanding natural phenomena, which utilizes numerical analysis and data mining of observations about natural phenomena, to complete awareness of the laws of nature. Many natural phenomena have multi-attributes, the covariance matrix between the attributes are important statistics reflect the interaction and interplay between features, lots of statistical analysis is done based on its [1,2].

The determinant value, corresponding to the covariance matrix of the observation, represents an important indicator of information dispersion degree and information aggregation degree, and the eigenvalues and eigenvectors of the covariance matrix are a simplified and comprehensive information corresponding to the original data, in general, large eigenvalue (eigenvector) contains more raw data information $[3,4]$.

In recent years, the research contents about the similarity figures matrix, the matrix decomposition and simplification, special matrix is very rich, and widely used. by constructing a linear transformation, this paper gives a method as analyzing the characteristics of matrix eigenvalues and eigenvectors, the corresponding determinant value is calculated, which is simple and feasible [5-9].

\section{Main results}

A mapping from a linear space to itself is called a transform.

Definition 1 A transform of linear space A is called a linear transform, if for arbitrarily $\alpha, \beta \in V$ and $k \in P$ (where $P$ is a number field), we have

$$
\begin{aligned}
& \mathrm{A}(\alpha+\beta)=\mathrm{A}(\alpha)+\mathrm{A}(\beta) \\
& \mathrm{A}(k \alpha)=k \mathrm{~A}(\alpha)
\end{aligned}
$$

Definition 2 Suppose $f(x)=a_{m} x^{m}+a_{m-1} x^{m-1}+\cdots+a_{0}$ is a polynomial in $P[x]$, A is a linear transform on $V$, a linear transform $f(A)$ is said to be a polynomial of $\mathrm{A}$, if

$$
f(A)=a_{m} A^{m}+a_{m-1} A^{m-1}+\cdots+a_{0} E
$$

Definition 3 Suppose $A$ is a linear transform on linear space $V$ and number field $P$, if there are a $\lambda_{0} \in P$ and a non-vanishing vector, makes

$$
\mathrm{A} \xi=\lambda_{0} \xi
$$

then $\lambda_{0}$ is called a eigenvalue of $\mathrm{A}, \xi$ is called the corresponding eigenvector of $\mathrm{A}$. 
In the view of geometry, after the linear transformation the direction of eigenvector always keep on the same line, when $\lambda_{0}>0$ the direction keep the same as before, as $\lambda_{0}<0$ the direction turn to opposite, and $\lambda_{0}=0$ the eigenvector is transformed to zero vector.

If $\xi$ is a eigenvector of the linear transformation A corresponding to $\lambda_{0}$, then $k \xi(k \neq 0)$ is a eigenvector of the linear transformation A corresponding to the same eigenvalue, which show that the eigenvectors are not uniqueness to a eigenvalue, but the eigenvalue is uniqueness for all eigenvectors.

Definition 4 Suppose $A_{n \times n}$ is a matrix on $P$, the determinant of matrix $\lambda E-A$

$$
|\lambda E-A|=\left|\begin{array}{cccc}
\lambda-a_{11} & -a_{12} & \ldots & -a_{1 n} \\
-a_{21} & \lambda-a_{22} & \ldots & -a_{2 n} \\
\ldots \ldots \ldots \ldots \ldots \ldots \ldots \ldots \ldots \ldots \ldots \ldots \ldots \\
-a_{n 1} & -a_{n 2} & \ldots & \lambda-a_{n n}
\end{array}\right| .
$$

is said as the characteristic polynomial of $A$, which is a $n$ order polynomial.

If $\lambda_{0}$ is a eigenvalue of linear transform $A$, then it must be a root of the characteristic polynomial, in turn, if there are some roots of the characteristic polynomial in the $P$, then the homogeneous equations (3) should have some nonzero solution, and the corresponding vectors are non-vanishing vectors. If $\left(x_{01}, x_{02}, \cdots, x_{0 n}\right)$ is a nonzero solution of equations (3), then the non-vanishing vector $\xi=\left(x_{01} \varepsilon_{1}+x_{02} \varepsilon_{2}+\cdots+x_{0 n} \varepsilon_{n}\right)$ satisfies equations (1), in other words, $\lambda_{0}$ and $\xi$ respectively are the eigenvalue and the corresponding eigenvector of $\mathrm{A}$.

Theorem 1 Suppose the matrix $A_{n \times n}$ has the eigenvalues as $\lambda_{1}, \lambda_{2}, \lambda_{3}, \cdots, \lambda_{n}$, Then $\lambda_{1}+\lambda_{2}+\cdots+\lambda_{n}=a_{11}+a_{22}+\cdots+a_{n n},|A|=\prod_{i=1}^{n} \lambda_{i}$.

Proof for

$$
|\lambda E-A|=\left|\begin{array}{cccc}
\lambda-a_{11} & -a_{12} & \ldots & -a_{1 n} \\
-a_{21} & \lambda-a_{22} & \ldots & -a_{2 n} \\
\ldots \ldots \ldots \ldots \ldots \ldots \ldots \ldots \ldots \ldots \ldots \ldots & \ldots \ldots \ldots \ldots \ldots \ldots \\
-a_{n 1} & -a_{n 2} & \ldots & \lambda-a_{n n}
\end{array}\right|
$$

its expanded form has a term which is the product of all element on the main diagonal line

$$
\left(\lambda-a_{11}\right)\left(\lambda-a_{22}\right) \cdots\left(\lambda-a_{n n}\right)
$$

In the rest term, the number of elements on the main diagonal is not more than $n-2$, the corresponding order of $\lambda$ is not more than $n-2$.

Therefore the items of $\lambda^{n}$ and $\lambda^{n-1}$ of the characteristic polynomial should only come from the product of all element on the main diagonal, they are

$\lambda^{n}-\left(a_{11}+a_{22}+\cdots+a_{n n}\right) \lambda^{n-1}$,

the characteristic polynomial is,

$|\lambda E-A|=\lambda^{n}-\left(a_{11}+a_{22}+\cdots+a_{n n}\right) \lambda^{n-1}+\cdots+(-1)^{n}|A|$.

let $\lambda=0$, we have

$|-A|=(-1)^{n}|A|$

According to the relationship of radicals and coefficients, we have

$\lambda_{1}+\lambda_{2}+\cdots+\lambda_{n}=a_{11}+a_{22}+\cdots+a_{n n}$, 
Which is called the trace of $A$, and

$|A|=\lambda_{1} \times \lambda_{2} \times \cdots \times \lambda_{n}$

Example 1 consider the matrix

$A=\left(\begin{array}{rrr}2 & -1 & 2 \\ 5 & -3 & 3 \\ -1 & 0 & -2\end{array}\right)$, we have
$|A-\lambda E|=\left|\begin{array}{ccc}2-\lambda & -1 & 2 \\ 5 & -3-\lambda & 3 \\ -1 & 0 & -2-\lambda\end{array}\right|=(2-\lambda)(-3-\lambda)(-2-\lambda)+\cdots=-(\lambda+1)^{3}$

then

$\lambda_{1}+\lambda_{2}+\lambda_{3}=a_{11}+a_{22}+a_{33}=-3, \lambda_{1}=\lambda_{2}=\lambda_{3}=-1$,

$|A|=-1$.

Example 2 consider the covariance matrix of agriculture sub-sector agriculture sub-sector economic growth rate in henna province between 2011-2014,

\begin{tabular}{|l|l|l|l|l|l|}
\hline & Farming & Forestry & Husbandr & Fishery & Services \\
\hline Farming & -0.569 & 1.364 & -0.878 & -0.459 & 1.355 \\
\hline Forestry & 0.223 & 1.621 & -0.423 & -0.753 & 1.255 \\
\hline Husbandry & 1.461 & 1.26 & -0.580 & 0.833 & -0.501 \\
\hline Fishery & -0.306 & 1.029 & 1.346 & -0.235 & 1.407 \\
\hline Services & 1.35 & -1.044 & 0.186 & 0.154 & 1.424 \\
\hline
\end{tabular}

$$
|A-\lambda E|=\left|\begin{array}{llllll}
-0.569-\lambda & 1.364 & -0.878 & -0.459 & 1.355 \\
0.223 & 1.621-\lambda & -0.423 & -0.753 & 1.255 \\
1.461 & 1.26 & -0.58-\lambda & 0.833 & -0.501 \\
-0.306 & 1.029 & 1.346 & -0.235-\lambda & 1.407 \\
1.35 & -1.044 & 0.186 & 0.154 & 1.424-\lambda
\end{array}\right|
$$

$=(-0.569-\lambda)(1.621-\lambda)(-0.58-\lambda)(-0.235-\lambda)(1.424-\lambda)+\cdots$.

We have

$\lambda_{1}=2.566, \lambda_{2}=1.567, \lambda_{3}=0.719, \lambda_{4}=1.147, \lambda_{5}=3.288 E-17$.

Therefore

$\lambda_{1}+\lambda_{2}+\lambda_{3}+\lambda_{4}+\lambda_{5}=a_{11}+a_{22}+a_{33}+a_{44}+a_{55}=1.661$,

$|A|=\lambda_{1} \times \lambda_{2} \times \lambda_{3} \times \lambda_{4} \times \lambda_{5}=1.40 E-17$.

\section{References}

[1] LI C. J.. Big data and new mind on statistics. Statistical Research Vol.31 (2014), p. 10-16.

[2] Lin H. M. , Du Z. F.. Some problems in comprehensive evaluation in the principal component analysis. Statistical Research Vol. 30 (2013), p. 25-31.

[3] QIU D.. A challenge of statictics in the age of big data. Statistical Research Vol. 31 (2014), p. $17-22$. 
[4] REN Z. Q., DONG L. Y.. Study on spatial variability of intra-county economics in liaoning province based on spatial statistical analysis. Economic Geography Vol. 30 (2010), p. 1435-1438.

[5] JIA Z.. Some applications of the equivalent standard form of matrix. Journal of Nanyang Teachers' College Vol.4 (2005), p. 29-33.

[6] MENG H. L., LIU X. X., WANG G. H.. A note on the equivalent canonical forms of matrices. Henan Science Vol. 30 (2015), p. 1072-1075.

[7] ZHANG G. L.. The solution to the numerical value of $\lambda$-matrix equivalent standard(1). Journal of Suzhou Railway Teachers College Vol. 13 (1996), p . 17-21.

[8] ZHANG G. L.. (1996) The solution to the numerical value of $\lambda$-matrix equivalent standard(2). Journal of Liaocheng Teachers College Vol.13 (1996), p. 17-24.

[9] ZHU R. K.. On equivalence standard form in graduate entrance examination questions. Study in College Mathematics Vol.13 (2013), p. 62-64. 